\title{
Lipoprotein Structure and Dynamics: Low Density Lipoprotein Viewed as a Highly Dynamic and Flexible Nanoparticle
}

\author{
Ruth Prassl and Peter Laggner \\ Additional information is available at the end of the chapter
}

http://dx.doi.org/10.5772/48145

\section{Introduction}

Low density lipoproteins (LDLs) are the principal transporter of cholesterol and fat in human blood. Circulating LDLs guarantee a constant supply of cholesterol for tissues and cells, whereas cholesterol is required for membrane synthesis, modulation of membrane fluidity and the regulation of cell signaling pathways. The function of LDL in metabolism is mediating by cellular uptake via receptor-mediated endocytosis followed by lysosomal degradation [1,2], and is strongly dependent on the lipid distribution, the structure of LDL particles and on the proper conformational orientation of apolipoprotein B100 (apoB100). Apo-B100 is the sole protein component of LDL being mainly located on the surface of LDL. Apart from their well established role as lipid transporter, LDL particles are intimately involved in the progression of cardiovascular diseases such as atherosclerosis or stroke, which are among the most prevalent causes of death in developed countries [3]. In particular, raised plasma levels of LDL are linked to an increased risk for disease. Moreover, dysregulations of LDL due to abnormalities in LDL structure have been identified as independent predictors of risk for coronary heart diseases [4,5]. LDL particles by themselves are highly heterogeneous in nature, varying in their buoyant densities, size, surface charge and chemical composition [6,7], and these biochemical characteristics determine the fate of LDL in the subendothelial space [8,9]. For example, small, dense LDL subclasses are more atherogenic than their light counterparts, which are more susceptible to modifications [5,10]. Modifications of LDL, primarily through oxidation, enzymatic degradation or lipolysis are the initiating factors in early atherosclerosis. In that case, LDL particles accumulate in the intima of the arterial wall where apo-B100 binds to proteoglycans of the extracellular matrix through ionic 
interactions. As a consequence, LDL becomes trapped in the subendothelium, where it is prone to oxidation processes, aggregation and fusion. Bioactive lipids, such as oxidized phospholipids, lysolipids or oxidized cholesterylester, are released from LDL particles, which are simultaneously non-specifically altered. A broad spectrum of diverse LDL particles with non-defined physicochemical properties is generated that, in turn, promotes a rapid uptake of these particles by macrophages to form foam cells [11]. This is one of the key steps in the progression of atherosclerosis. Today, atherosclerosis is known to be a chronic inflammatory disorder of the blood vessels and recognized as a prevailing cause of cardiovascular disorders, the leading causes of morbidity and mortality worldwide [12]. Since the early initiation of atherosclerosis strongly depends on the metabolism of LDL, which is predominantly triggered by molecular characteristics of LDL, it is of paramount biomedical importance to explore structural features of LDL particles in great detail. However, mostly due to the complex nature of LDL particles many questions concerning molecular details are still unanswered.

This article will review our current knowledge on the structure and dynamics of LDL particles. In fact, several recent studies revealed that the molecular organisation and dynamics of LDL core lipids, in close relationship to the intrinsic dynamics of LDL surface components, control not only the metabolism of lipids in humans, but determine the role of LDL in the pathogenesis of cardiovascular diseases. In this article, we will give a short historical review on LDL structure and then present prevailing concepts on the selforganisation of LDL. Special emphasis will be paid to dynamic features of LDL particles. In particular, we will discuss the interplay between structure and dynamics in more detail. Finally, we will give an outlook to promising future strategies to clarify the molecular structural details of LDL and how to exploit LDL nanoparticles for medical needs.

\section{Molecular architecture of LDL}

LDLs are composed of lipids and protein, which assemble to form a supramolecular complex with a molecular mass exceeding 2.5 - 3.0 million Da and involving 2000 to 3000 lipid molecules. Thus, LDL particles are commonly described as micellar complexes, macromolecular assemblies, self-organized nanoparticles or microemulsions. Regardless of diverse definitions, it is generally accepted that assembled LDL particles are organized into two major compartments, namely an apolar core, comprised primarily of cholesteryl esters (CE), minor amounts of triglycerides (TG) and some free unesterified cholesterol (FC). The core is surrounded by an amphipathic outer shell. This shell is composed of a phospholipid (PL) monolayer containing the larger part $(>2 / 3)$ of the FC molecules and one single copy of apo-B100, which is one of the largest known monomeric glycoproteins [13]. Figure 1 provides an overview on characteristic properties of LDL together with a schematic presentation of an LDL particle. Since molecular interactions between different kinds of lipids have turned out to be highly complex, it is almost impossible to separate the surface 
and core regions exactly from each other. Accordingly, in some recent reports an additional hydrophobic interfacial layer composed of phospholipid acyl chains, FC, some CE molecules and hydrophobic protein domains is defined. This description takes account for the interplay between neutral core lipids and the surface layer [14].

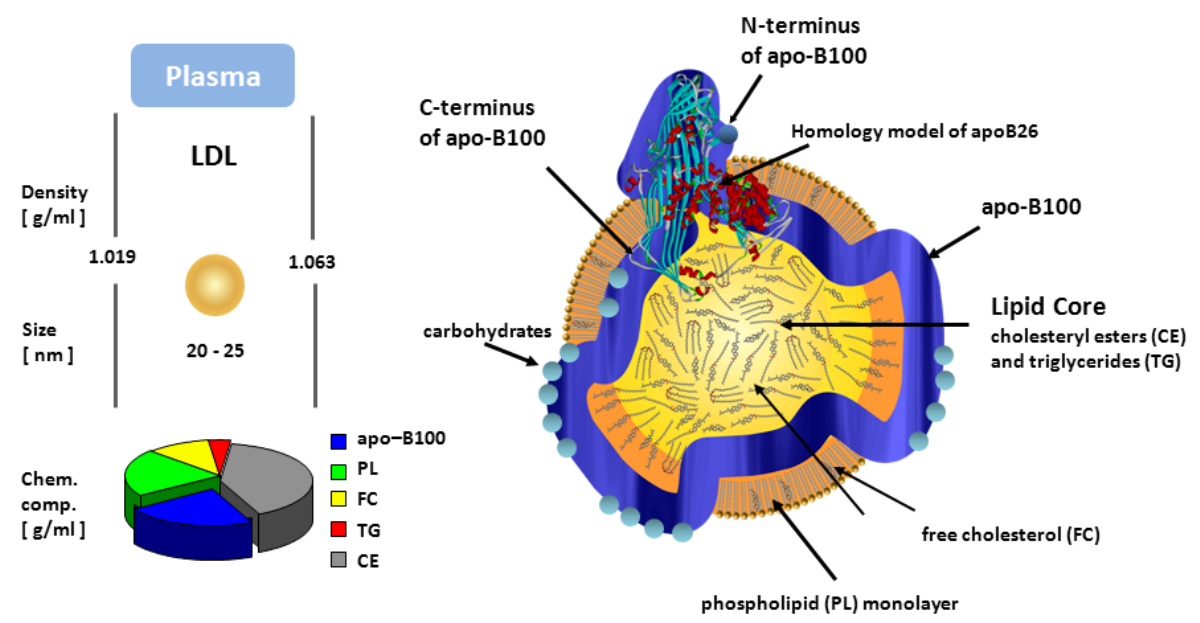

Figure 1. Molecular organisation of LDL. LDL particles are isolated from human plasma within a defined density range. Their particle size varies between 20 to $25 \mathrm{~nm}$. LDLs are built up by a hydrophobic lipid core of cholesterylester (CE) and triglyceride (TG) molecules, which make up more than $40 \%$ of particle mass surrounded by a phospholipid (PL) monolayer corresponding to about $20 \%$ of particle mass. Varying amounts of free cholesterol (FC) are incorporated in the shell and the core regions. One single copy of apo-B100 (550 kDa) is embedded in the surface monolayer, partially penetrating the core and covering about 40 to $60 \%$ of the surface area. The carbohydrate moieties are distributed along the protein chain and are surface exposed. The N-terminal end of apo-B100 (about $26 \%$ of total) is hydrophilic and shows a high homology to lamprey lipovitellin. The C-terminal end was shown to be located close to the $\mathrm{N}$-terminus.

Since LDL particles are highly heterogeneous, especially with respect to the chemical composition of the core lipids, the actual size of LDL particles varies between 20 to $25 \mathrm{~nm}$, with an average particle diameter of about $22 \mathrm{~nm}$. This intrinsic heterogeneity allows a subdivision of LDLs into distinct highly homogeneous LDL subspecies, which are identified on the basis of their hydrated densities, which normally lies between the extremes of $\mathrm{d}, 1.019$ and $1.063 \mathrm{~g} \mathrm{ml}^{-1}$ [15]. These subspecies also differ in their physicochemical characteristics, receptor binding affinity [16], susceptibility to oxidative modifications [17,18], and in their atherogenic behaviour. Following these lines, it is important to consider LDL as a flexible construct, which needs to respond to changing environmental conditions during lipid exchange. Hence, during particle remodelling, apoB100 and the surface PL molecules have to rearrange to compensate for changes in the 
surface area and surface pressure [6]. It is known, that apo-B100 predominantly resides on the surface of LDL and displaces PL molecules, concomitantly changing the diffusion and order parameter of lipids as shown in a recent near atomistic simulation study [19]. Based on simple geometrical considerations taking into account the surface PL monolayer (about 700 PL molecules) with an average area per lipid of $0.65 \mathrm{~nm}^{2}$ and an LDL particle diameter of $22 \mathrm{~nm}$, large parts of the surface layer must be covered by the protein to avoid unfavourable hydrophobic contacts. In support of these considerations, a loose surface packing of PL molecules was derived from molecular dynamics simulations [19]. This low surface pressure enables hydrophobic amino acid regions of apo-B100 to penetrate into the interfacial regions, predominantly formed by the acyl chains of PLs. Consequently, apo-B100 might interact more readily with the neutral core lipids, and indeed it was shown that some of the CE molecules align along the $\beta$-sheet structures of apo-B100 [20], thereby driving CE molecules to the surface, where they become part of the interfacial layer. Particularly noteworthy is the fact that the lipids within the interfacial layer are not homogeneously distributed but form local microenvironments [14]. More precisely, two nanodomains were identified, one rich on sphingomyelin and FC, the other one rich in phosphatidylcholine and poor in FC. The latter was shown to be associated more closely with apo-B100 [21]. Even though, one has to keep in mind that these domains are not static or confined in size and number and co-determine the intrinsic dynamics of LDL. Based on these types of findings, it seems reasonable to suggest that variations in the molecular organisation of lipid/apo-B100 impact the structure of LDL, and have to be considered to act as physiological determinants of LDL function.

\section{Structural models of LDL}

Our present understanding of the structure of LDL particles has emerged from the concerted application of different physico-chemical techniques with early ground-breaking findings derived from neutron- or X-ray small angle scattering data [22-25] complemented by results from negative staining electron micoscopic (e.m.) [26,27] and spectroscopic techniques [28,29]. For comprehensive reviews on different biophysical studies applied on LDL species see refs. [30,31]. In recent years structural investigations using cryo-e.m. reconstruction techniques have become prevalent and with time 3-dimensional models with improved resolution were presented [32-37]. While in earlier studies LDLs are described as quasi-spherical particles, later studies presented a new view of the overall particle structure displaying an oblate elliptical particle shape. Moreover, recent 3D-images show convincing data that LDL can be considered as discoidal-shaped particle with two flat surfaces on opposite sides. In this model, apo B100 encircles LDL at the edge of the particle, while the PL monolayer is rather located at the flat surfaces which are parallel to the CE layers in the core $[36,37]$. To get a better impression of what LDL looks like in a structure map obtained by 3D-reconstruction from cryo-e.m, we show some images in Figure 2 revealing the surface density distribution on LDL. It has to be stated that this model strictly holds true for LDL particles with the core lipids being in a frozen liquid-crystalline state. 

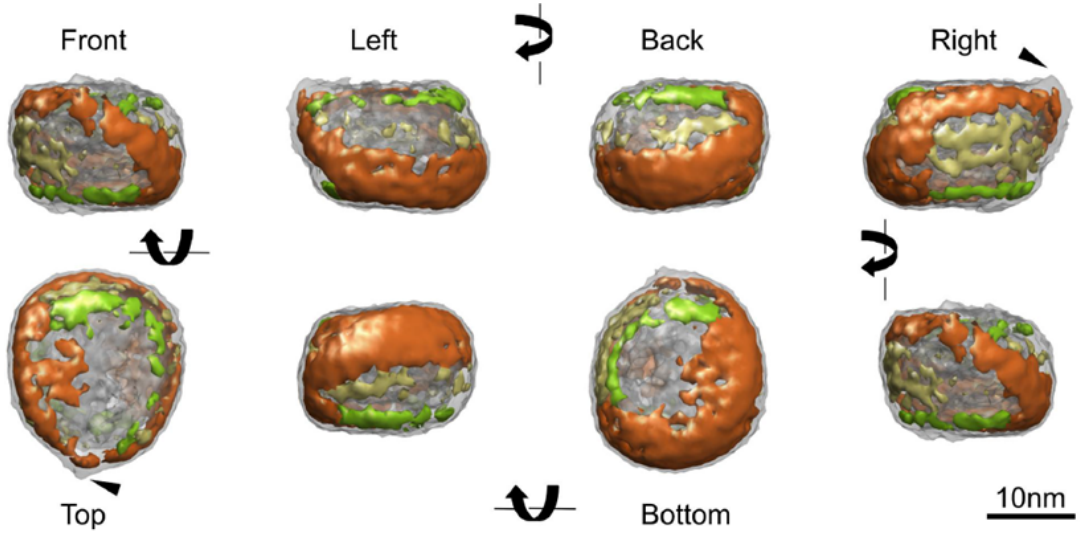

Figure 2. Density distribution at the surface of LDL. The 3D-density map derived from cryo e.m. images by reconstitution reveals the oblate overall particle shape of LDL shown in gray. The overlaid high density regions represent the backbone of apo-B100, colored in orange. The belt surrounds the particle to form an enclosed circle. The second group of high density regions (green) contours the rims and complements the backbone enclosing lower-density regions. The high density regions on the sidewall (yellow) are structures extending from the backbone. A knob-like protrusion is visible at the pointed end (indicated by triangles in the right and top views). The 3D-map is turned $90^{\circ}$ in each frame. Reprinted with permission from ref. [37].

\section{Core lipid packing and lipid phase transition}

Despite of compositional heterogeneity, LDL particles share one common feature: the CE molecules in the core undergo a structural transition from an ordered liquid-crystalline phase to a fluid oil-like state as function of temperature and chemical composition [38]. More precisely, the actual transition temperature, which is close to body temperature, is inversely correlated to the content of triglycerides within the lipid core [22,39]. Based on these characteristics, several models for CE packing have been suggested including a spherical concentric layer model derived primarily from X-ray and neutron scattering data [40,41]. More recently, the concept of a flat lamellar structure came up. This model is derived from singleparticle reconstructions from cryo-e.m. images of LDL in vitreous ice [32,34]. An ordered three-layer internal lamellar structure with a distance of about 3.6. $\mathrm{nm}$ between the single lamellae was reported [32], in agreement with repeat distances derived from X-ray scattering patterns for LDL below the transition temperature. While these images were observed for LDL particles being in the liquid crystalline phase before snap-freezing, diverse results were reported for LDL particles frozen from a state above the phase transition temperature $[42,43]$. One plausible explanation for these discrepancies might be that the melting rate of the core lipids proceeds extremely fast. It has been shown that the physical state of core lipids changes within milliseconds [44]. This fast kinetics has caused experimental difficulties for long time, however, a recent experimental approach by speeding up freezing allowed to trap the lipids in the molten state [45]. The authors report on a co-existing phase of layered and broken shells for LDL particles, which are shock-frozen in a state above the phase transition. This is the first 
time to visualize the nucleation process of CEs within LDL. Most interesting, the images indicate intermediate states between the order/disorder phase transition. Figure 3 shows the dynamic model of the core CE packing during the phase transition and gives a comparison of the internal features of reconstructed 3D-volumes of LDL.
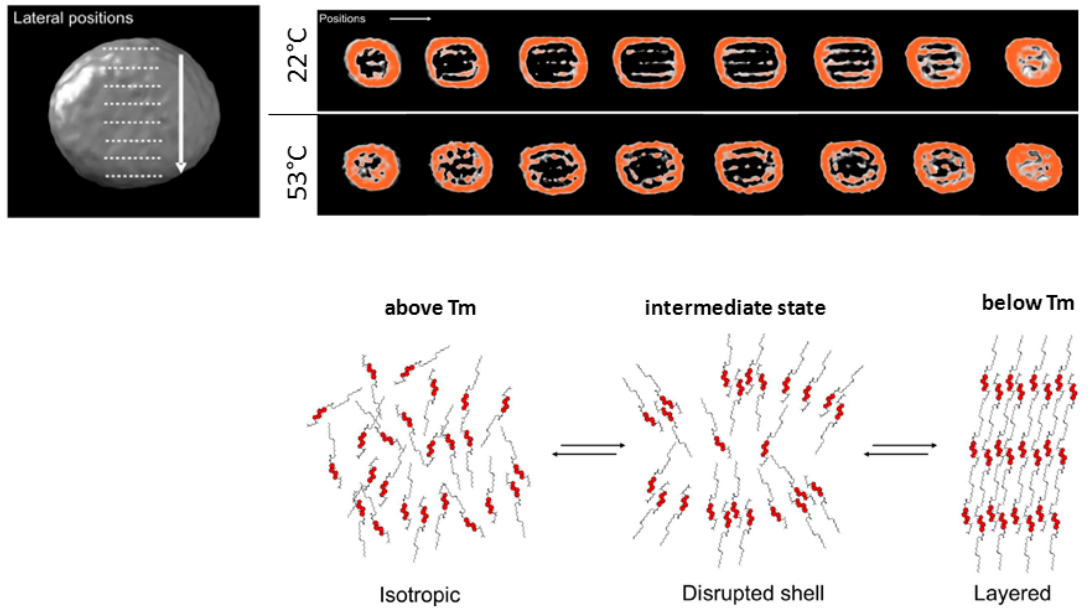

Figure 3. Schematic picture of the dynamic model of LDL core lipid packing during the phase transition. Comparision of the internal features of the reconstructed 3D-volume of LDL snap-frozen from below $\left(22^{\circ} \mathrm{C}\right)$ and above $\left(53^{\circ} \mathrm{C}\right)$ the phase transition temperature $(\mathrm{Tm})$. Samples prepared from $22^{\circ} \mathrm{C}$ show a layered organisation while samples prepared from $53^{\circ} \mathrm{C}$ reveal a disorded shell like structure, which is concentric to the surface. Note, the overall shape of LDL has also changed slightly. The lower panel shows a hypothetical model for the core lipid packing depicting the dynamic process of the core lipid phase transition upon cooling from isotropic to layered passing through an intermediate state. Modified with permission from ref. [45].

In summary, it seems reasonable to argue that both the overall shape and core lipid packing of LDL particles are highly sensitive to changes in temperature and lipid composition. Indeed, this newly proposed patch nucleation behavior permits the temporary formation of local molecular microenvironments as suggested previously by our group in terms of trigylceride segregation [46]. In the next paragraph we will address some interesting questions in support of above hypotheses.

Does a lipid microphase separation occur in LDL particles as a function of the relative core content of CE and TG?

As already mentioned, the transition temperature correlates with the lipid composition, however, a discontinuity in the concentration dependence was observed [46]. A break in the concentration dependence of a transition temperature in a mixed lipid system constitutes an index for the existence of a phase separation at the break point. In isolated triglyceride cholesteryl ester systems no indication of a phase separation at similar compositions was found [39,47]. It appears therefore, that structural constraints within the LDL particle 
determine this effect. Experimental data provide evidence that at low TG content (below $12 \%)$ the TG molecules separate into distinct hydrophobic nanoenvironments while the CEs form a smectic liquid crystalline layer. With increasing TG content the thermal stability of the CE layer is decreased by intermixing with TG [46]. This hypothesis implies that the TGrich fluid nanodomains can serve as a reservoir for lipophilic minor constituents, such as vitamins (tocopherol, carotenoids etc.) below the phase transition. The local concentration of these antioxidants and hence their efficiency in scavenging lipophilic free radicals is higher than if they were dissolved in the bulk volume of total apolar lipids. At the same conditions the CE molecules are strongly immobilized and the intracellular degradation of LDL is decelerated [48], equally the activity of lipid transfer proteins is diminished [49,50]. Based on these considerations it is tempting to speculate that circulating LDL, as a consequence of the variation in blood temperature, periodically undergoes a thermal transition resulting in a transient increase in the local core concentration of minor constituents [46]. Here, it should be emphasized that a periodic redistribution of lipophilic solutes, and also for example of drugs, into the confined LDL core volume could represent an attractive approach to the modulation of biochemical reactions, which would not occur at sufficient rates under the normal conditions of relative concentration. Studies along these lines could indeed verify the long missing physiological role of the thermal LDL transition.

Can LDL structure follow quasi-isothermal changes in blood temperature during its circulation, or does it remain adiabatically metastable in the molten-lipid state?

In order to provide evidence to answer this question we have applied time resolved X-ray scattering experiments using a high flux synchrotron generated X-ray beam. Thus, we have been able to trigger the thermal transition in either direction (heating and cooling) simultaneously monitoring associated structural changes in sub-second time intervals. With our special instrumental setup we managed to evaluate the kinetics of core-transition by Tjump and T-drop experiments [44]. We found that the melting transition proceeds faster than 10 milliseconds indicating that thermal-induced lipid reorganisation takes place at the time scale of blood circulation. As the velocity of blood-flow can be as low as $0.3 \mathrm{~mm} / \mathrm{s}$ in peripheral blood capillaries the residence time for LDL particles in cooler regions of the body can be several seconds. Consequently, LDL can easily follow periodic temperature changes during blood circulation and assist the redistribution of lipophilic constituents within its core nanodomains forming fluid defect zones. For biomedicine, this strengthens the hypothesis that the core lipids of LDL not only act as passive chemical substrates in metabolism, but that their physical state within the LDL nanoparticles has the potential to control their metabolic fate in normal and atherosclerotic cholesterol transport.

Does the core lipid transition have a physiological meaning ?

Despite its occurrence conspicuously close to blood temperature and the variation of the transition temperature of LDL among different subjects, no clear evidence for a physiological or patho-biochemical role of this transition has so far been found. It is now generally accepted in literature that the rearrangement of the core lipids also affects the overall structure and shape of the LDL particle. Morphological changes in turn can impact receptor-binding activity as well as the action of lipid hydrolyzing enzymes. Equally, the 
susceptibility of LDL particles to oxidative modifications and lipid peroxidation might be correlated to temperature [18]. As oxidized LDL play a crucial role in the pathogenesis of atherosclerosis, any contribution to the comprehension of antioxidant efficiency may be of therapeutic potential $[2,51]$, further pointing to the physiological relevance of the lipid core organisation. However, this vital question still remains unanswered.

\section{Apo-B100 is a flexible string wrapped around the surface of LDL}

As already indicated above, the physicochemical state of the core lipids is intimately related to the structure and dynamics of the particle surface, which consists of about 700 phospholipid molecules and one single copy of apo-B100. Apo-B100 is a huge glycoprotein and its polypeptide chain consists of 4536 amino acid residues with an estimated molecular mass of about $550 \mathrm{kDa}$ for the glycosilated form [52,53]. Apo-B100 is a single chain protein with a total contour length of about $70 \mathrm{~nm}$ [54] and can be viewed as a highly flexible molecular string composed of single domains [20]. Five consecutive domains were identified based on secondary structure elements representing the main conformational motifs of apo-B100. The single domains are connected by flexible interdomain linker regions, which allow relative movements of domains to each other. The feasibility of such motions was shown in a low resolution model of detergent solubilized apo-B100, which was derived from small angle neutron scattering data [55]. In this model, compact rigid domains are visible being connected by flexible interdomain linkages, which possess a substantial degree of freedom in their spatial orientation. A hypothetical spatial arrangement of the apo-B100 molecule on a spherical LDL particle was created after assigning the secondary structure elements, which were deduced from a secondary structure prediction, to the surface of apo-B100 (Figure 4). Likewise, the averaged surface shape of the 3D-model would allow for variations in the thickness of the apoB100 molecule by about $1 \mathrm{~nm}$. Such variations are most likely required to compensate for changes in the surface area upon lipid exchange and particle shrinking during endogeneous lipoprotein conversion from very low density lipoprotein (VLDL) to LDL.

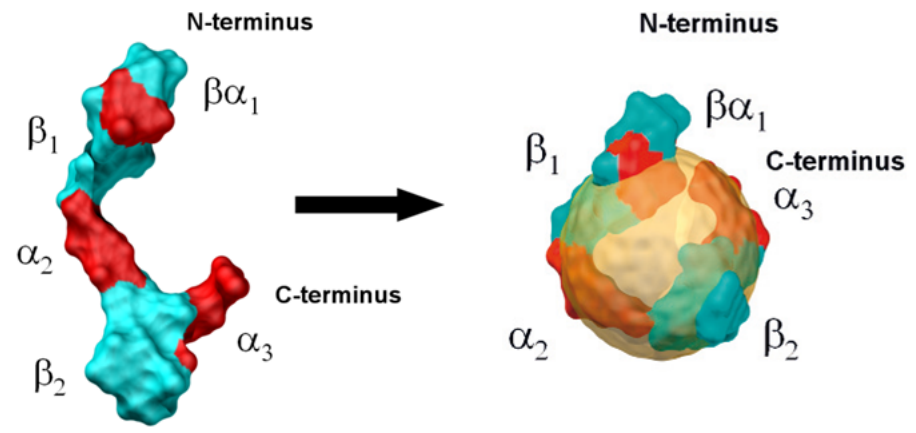

Figure 4. Reconstituted low resolution model of lipid-free apo-B100 derived from small angle neutron scattering data. Apo-B100 shows an elongated arch-like morphology indicating single domains and mobile less defined linker regions. A hypothetical model of a spherical LDL particle after superposition of the structural model of apo-B100 is shown (adapted from ref. [55]). Secondary structure modules are assigned to the surface after a secondary structure prediction was performed. The results nicely correspond to the pentapartite model suggested by [20]. 
Concerning the topology of apo-B100 on the surface of LDL the most detailed information is obtained from cryo-e.m. images (see also Figure 2). Chatterton et al. were among the first to visualize apo-B100 as string circumventing LDL, and to report on mapped epitopes of apoB100 distributed over one hemisphere of the LDL particle [56,57]. Recent single particle 3Dreconstructions from immuno cryo e.m. images delineated a more accurate picture of apoB100 revealing a looped topology of the protein backbone with distinct epitopes identified along the protein chain. According to this model, epitopes in the LDL receptor binding domain are located on one side of LDL, whereas epitopes located in the N-terminal and Cterminal domains are in close vicinity to each other on the opposite side of LDL [36]. In addition, a prominent protrusion is visible in the images at the pointed end of the particle. A similar knob-like region was apparent in the low resolution model of lipid-free apo-B100 shown in Figure 4. This protrusion most probable represents the non-lipid associated globular N-terminal domain of apo-B100, which shows a high homology to lamprey lipovitellin [58]. Except for the N-terminal domain, little is known about the molecular organisation of the structural motifs, whose amphipathic nature determine lipid association. However, to evaluate lipid-protein interactions physical parameter like interfacial elasticity or molecular dynamics have to be considered. In this context, it was suggested that the hydrophobic $\beta$-sheet domains of apo-B100 act as elastic lipid anchors, whereas the amphipathic $\alpha$-helical domains respond rapidly to changes in surface pressure $[59,60]$. In any case, it can be assumed that alterations in the adsorption and penetration depth of apoB100 in the phospholipid monolayer and in the lipid core are accompanied by structural rearrangements of the domains and changes in the orientation of the domains relative to each other. In the course of such elastic motions, intramolecular rearrangements are likely to alter the overall hydrophobicity and surface activity of single protein domains. These modifications not only affect lipid-protein interaction, but are equally important for molecular and cellular recognition of apo-B100.

\section{Apo-B100 containing lipoproteins are very soft and flexible}

LDL particles are formed in the circulation by lipolytic conversion of TG-rich VLDL particles. This enzyme mediated endogenous transformation is accompanied by an extensive shrinking in particle size from about 50-80 $\mathrm{nm}$ for VLDL to $\sim 20 \mathrm{~nm}$ for LDL. In the course of remodelling, apo-B100 remains bound to its nanocarrier stabilizing the lipid assembly by maintaining structural integrity. To accomplish this, apoB100 has to become more condensed or relaxed depending on the lipid packing density. Likewise, this dynamic process is modulated by the molecular mobility of the surrounding microenvironment. To test for this hypothesis we have recorded temperature dependent molecular motions in VLDL and LDL particles using elastic incoherent neutron scattering [61]. With this technique, motions in the nano- to picosecond time scale can be recorded. The calculated dynamic force constants are a direct measure for the resilience of the particles. The results show that at physiological temperatures VLDL particles are very soft, elastic and mobile as compared to LDL, which is more rigid (see Figure 5). This observation supports the notion that apo-B100 in VLDL is loosely packed at the interface covering a large surface area with 
low interfacial surface tension [59]. During particle conversion from VLDL to LDL, however, the relative number of surface molecules increases and a higher molecular packing density leads to a compression of the lipid anchored protein regions and an overall stiffening of the LDL particle [60].

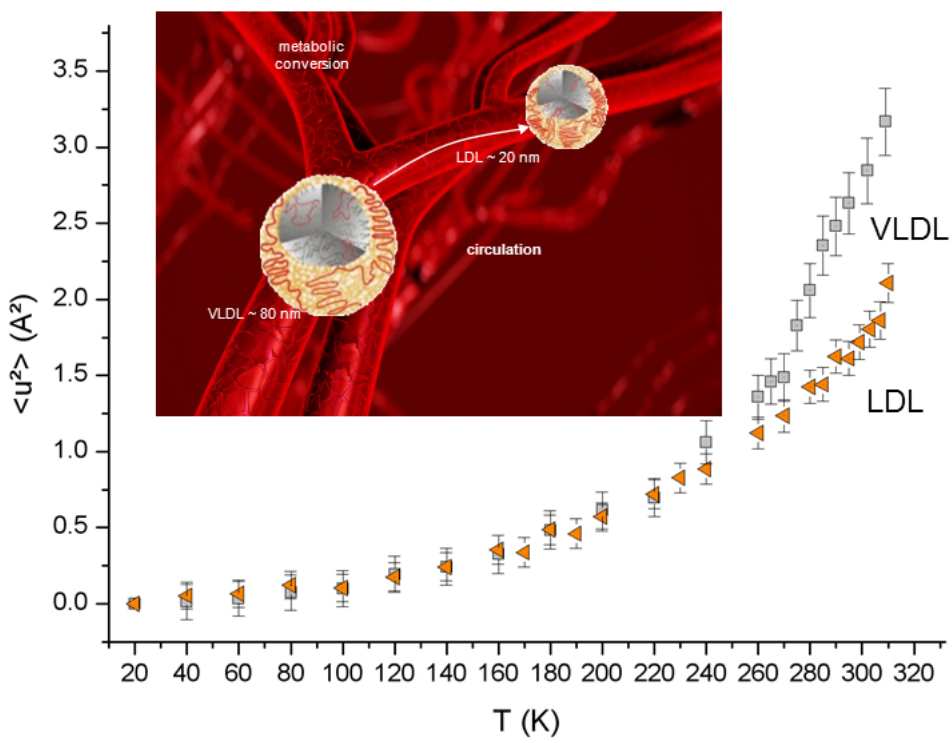

Figure 5. Molecular motions in LDL and VLDL. Elastic temperature scans are recorded with elastic incoherent neutron scattering. The mean square thermal fluctuations $\left.\left(<\mathrm{u}^{2}\right\rangle\right)$ are shown as function of temperature. The molecular resiliences are derived from the slopes in the curves. It is seen that VLDL has an increased motion at elevated temperatures compared to LDL. Parts of this figure are reproduced, with permission, from ref. [60].

To conclude, the intrinsic conformational flexibility and elasticity of apo-B100 containing lipoprotein particles is most likely critical for specific affinities of lipoproteins to receptors, antibodies or enzymes. Moreover, it would seem that the susceptibility of lipoproteins to oxidative modifications and hence their atherogenicity is influenced by their dynamic nature.

\section{LDLs are flexible nanotransporters circulating in blood}

In the search for new and improved therapeutics, the field of nanomedicine dealing with functionalized nanoparticles for molecular imaging and therapy is rapidly emerging. Nanoparticles offer new opportunities to transfer active substances directly to the diseased site in the body. By additional surface coatings or functionalizations, the properties of nanoparticles can be tuned to specific needs. Within the last two decades, a variety of artificial nanoparticles have been designed for targeted delivery of drugs or contrast agents. Many of these nanoconstructs are developed for cancer therapy taking advantage of the 
leaky vasculature of tumours. Apart from tumour targeting, increasing efforts are devoted to the treatment and imaging of atherosclerotic plaques (for a recent review see ref. [62]). Over time, a broad and versatile nanoparticle platform was created in which liposomes and biodegradable polymers have turned out to be the most promising candidates. It is important to mention that several nanomedicine products have already been established on the market and numerous products are successfully applied in clinical trials [63]. However, inherent problems of nanoparticles are biocompatibility and low stability in vivo, since most nanoparticles become rapidly cleared by the reticuloendothelial system. In contrast to artificial systems, lipoproteins are naturally occurring nanoparticles evading recognition by the body's immune system. Hence, lipoproteins are excellent candidates with attractive properties to be considered as molecular transporters. A great advantage of LDL over other nanoparticles is the fact that LDL particles stay in circulation for several days, and are not cleared immediately by the mononuclear phagocyte system of the liver and spleen. The average lifetime of an LDL particle is 2-3 days and this time span is about three times longer as reported for long-circulating liposomes, currently applied in chemotherapy [64]. It was recognized that certain tumor cells overexpress LDL receptor, however, the targeting specificity is limited as the LDL receptor is ubiquitously expressed throughout the body, most prominent in the liver. However, using apo-B100 as inherent targeting sequence the enhanced circulation times in blood enable drug-loaded LDL particles to bind to specific receptors exposed on the surface of e.g. tumor or atherosclerotic plaque. Once recognized by the receptor, the functionalized LDL particles become internalized, accumulate in the tissue and exert an enhanced effect (reviewed by [65]). The intrinsic targeting properties of LDL to atherosclerotic plaques are already utilized for early diagnosis and detection of atherosclerotic lesions by different imaging modalities (for reviews see refs. [66,67]). However, to modify lipoprotein particles for medical purposes, care has to be taken not to compromise essential biophysical and structural features of LDL with the goal to preserve the biological activity. In general, there are several possibilities to create multifunctionalized lipoprotein particles. Some representative examples are shown in the scheme in Figure 7. One possibility is to load hydrophobic drugs (e.g. chemotherapeutics, antibiotics, vitamins, signal emitting molecules or small nanocrystals) in the lipophilic inner core of LDL. This can be accomplished by different techniques including lyophilisation, solvent evaporation and reconstitution procedures [68,69]. However, LDL particles can not be reconstituted so easily and remote drug/contrast agent loading into native lipoprotein particles is still a tedious approach currently not being standardized. Amphiphilic substances (drugs or marker molecules) or fatty acid modified chelator complexes can be incorporated in the PL monolayer [70,71]. This has successfully been done in numerous biophysical studies and for diagnostic purposes. Finally, the surface of LDL can be modified by protein labeling. This is done by covalent attachment of substances to the lysine and cysteine amino acid residues of apo-B100. Such substances include fluorophores, radionuclides or metal ions for molecular imaging [65]. Alternatively, targeting sequences (e.g. folic acid) can be coupled to apo-B100 with the purpose to reroute LDL to alternate receptors, which, in case of folate, are more specifically expressed in tumor cells [72]. 


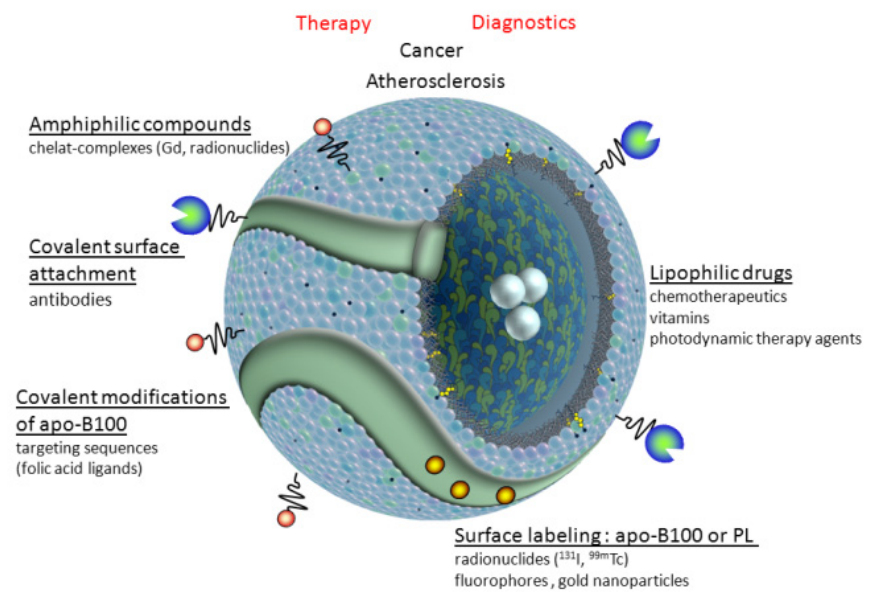

Figure 6. Scheme giving some examples of how LDL particles can be modified to act as natural endogenous nanoparticles for targeted drug delivery or multifunctional molecular imaging.

To construct lipoprotein mimetic particles, also referred to as lipoprotein related particles, artificial lipoprotein particles have to be reassembled from individual lipid and protein entities. This approach was highly successful for high density lipoproteins using apo-AI mimetic peptide sequences [73]. For LDL, this approach was not pursued yet and will be much more complicated considering the complex dynamic nature of apo-B100.

Over the last few years, a promising nanoparticle platform was established, which exploits the endogenous properties of natural lipoproteins being non-toxic, non-immunogenic and biodegradable. Although this platform still offers vast potential for improvements, first promising results in enhanced multimodal imaging of tumors and atherosclerotic plaques are achieved giving hope that further endeavors to combine diagnostics and personalized therapeutics will also be successful.

\section{Conclusions and future directions}

The intrinsic flexibility and dynamics of LDL lipids and protein in conjunction with the inherent compositional heterogeneity of LDL particles has hitherto hampered successful structure determinations at atomic level. Recent technological developments, however, allowed to restore characteristic structural features of individual LDL particles at low resolution. In particular, using cryo e.m. 3D-reconstruction techniques several groups have succeeded in imaging morphological and topological details of LDL to a resolution limit of approximately $2 \mathrm{~nm}$ [34-36]. Now, new concepts will be needed to make further progress in the development of high resolution models of LDL. One promising way is to put stronger emphasis on protein crystallography in combination with computational modelling and molecular dynamics simulations. X-ray crystallography apprears to be a hopeless pursuit 
with heterogeneous and flexible particles like LDL. Nevertheless, our earlier attempts of crystallisation have been partially successful [74]. Additional efforts, however, have to be focussed on the stabilization of apo-B100 in a more rigid state, perhaps by co-crystallisation with monoclonal antibodies. An alternative way ahead would be to work with lipid-free apo-B100 stabilized by detergent-mimetic systems, e.g. amphipathic designer peptides, or to proceed with truncated fragments of apo-B100.

At present there is still a deficit in our knowledge concerning the molecular lipid trafficking mechanisms of LDL. To know the atomic structure of LDL, in particular of apo-B100, may well contribute to a better understanding of biologic aspects of cardiovascular diseases, especially with respect to future strategies towards rational pharmaceutical interventions.

\section{Author details}

Ruth Prassl and Peter Laggner

Institute of Biophysics and Nanosystems Research, Austrian Academy of Sciences, Graz, Austria

\section{Acknowledgement}

This manuscript is based in part upon work supported by the Austrian Science Fund under grant number P-20455.

\section{References}

[1] Brown MS and Goldstein JL (1976) Receptor-mediated control of cholesterol metabolism. Science 191: 150-154.

[2] Steinberg D, Parthasarathy S, Carew S, Khoo JC, Witztum JL (1989) Beyond cholesterol. Modifications of low-density lipoprotein that increase its atherogenicity. New Engl.J.Med. 320: 915-924.

[3] Lusis AJ (2000) Atherosclerosis. Nature 407: 233-241.

[4] Packard C, Caslake M, Shepherd J (2000) The role of small, dense low density lipoprotein (LDL): a new look. Int.J.Cardiol. 74 Suppl 1: S17-S22.

[5] Packard CJ (2006) Small dense low-density lipoprotein and its role as an independent predictor of cardiovascular disease. Curr.Opin.Lipidol. 17: 412-417.

[6] McNamara JR, Small DM, Li ZL, Schaefer EJ (1996) Differences in LDL subspecies involve alterations in lipid composition and conformational changes in apolipoprotein B. J.Lipid Res. 37: 1924-1935.

[7] Chapman MJ, Guerin M, Bruckert E (1998) Atherogenic, dense low-density lipoproteins. Pathophysiology and new therapeutic approaches. Eur.Heart J. 19 Suppl A: A24-A30.

[8] Pentikainen MO, Oksjoki R, Oorni K, Kovanen PT (2002) Lipoprotein lipase in the arterial wall: linking LDL to the arterial extracellular matrix and much more. Arterioscler.Thromb.Vasc.Biol. 22: 211-217. 
[9] Skalen K, Gustafsson M, Rydberg EK, Hulten LM, Wiklund O, Innerarity TL, Boren J (2002) Subendothelial retention of atherogenic lipoproteins in early atherosclerosis. Nature 417: 750-754.

[10] Hurt-Camejo E, Camejo G, Sartipy P (2000) Phospholipase A2 and small, dense lowdensity lipoprotein. Curr.Opin.Lipidol. 11: 465-471.

[11] Williams KJ and Tabas I (2005) Lipoprotein retention--and clues for atheroma regression. Arterioscler.Thromb.Vasc.Biol. 25: 1536-1540.

[12] Hansson GK and Hermansson A (2011) The immune system in atherosclerosis. Nature Immunology 12: 204-212.

[13] Kostner, G. M. and Laggner, P. (1989) in Human Plasma Lipoproteins - Clinical Biochemistry, Principles, Methods, Applications 3 (Fruchart, J. C. and Shepherd, J., eds.), pp. 23-54, Walter de Gruyter, Berlin - New York.

[14] Hevonoja T, Pentikainen MO, Hyvonen MT, Kovanen PT, Ala-Korpela M (2000) Structure of low density lipoprotein (LDL) particles: basis for understanding molecular changes in modified LDL [In Process Citation]. Biochim.Biophys.Acta 1488: 189-210.

[15] Chapman MJ, Laplaud PM, Luc G, Forgez P, Bruckert E, Goulinet S, Lagrange D (1988) Further resolution of the low density lipoprotein spectrum in normal human plasma: physicochemical characteristics of discrete subspecies separated by density gradient ultracentrifugation. J.Lipid Res. 29: 442-458.

[16] Nigon F, Lesnik P, Rouis M, Chapman MJ (1991) Discrete subspecies of human low density lipoproteins are heterogeneous in their interaction with the cellular LDL receptor. J.Lipid Res. 32, 1741-1753.

[17] Dejager S, Bruckert E, Chapman MJ (1993) Dense low lipoprotein subspecies with diminished oxidative resistance predominate in combined hyperlipidemia. J.Lipid Res. 34, 295-308.

[18] Schuster B, Prassl R, Nigon F, Chapman MJ, Laggner P (1995) Core lipid structure is a major determinant of the oxidative resistance of low density lipoprotein. Proc.Natl.Acad.Sci.USA 92: 2509-2513.

[19] Murtola T, Vuorela TA, Hyvonen MT, Marrink SJ, Karttunen M, Vattulainen I (2011) Low density lipoprotein: structure, dynamics, and interactions of apoB-100 with lipids. Soft Matter 7: 8135-8141.

[20] Segrest JP, Jones MK, De Loof H, Dashti N (2001) Structure of apolipoprotein B-100 in low density lipoproteins. J.Lipid Res. 42: 1346-1367.

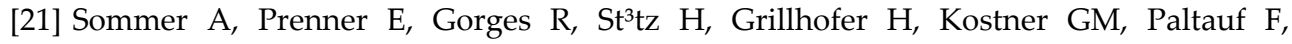
Hermetter A (1992) Organization of phosphatidylcholine and sphingomyelin in the surface monolayer of low density lipoprotein and lipoprotein(a) as determined by timeresolved fluorometry. J.Biol.Chem. 267: 24217-24222.

[22] Atkinson D, Deckelbaum RJ, Small DM, Shipley GG (1977) Structure of human plasma low-density lipoproteins: Molecular organization of the central core. Proc.Natl.Acad.Sci.USA 74: 1042-1046.

[23] Laggner P, Degovics G, Müller KW, Glatter O, Kostner GM, Holasek A (1977) Molecular packing and fluidity of lipids in human serum low density lipoproteins. Hoppe-Seyler's Z.Physiol.Chem. 358: 771-778. 
[24] Laggner P and Kostner GM (1978) Thermotropic changes in the surface structure of lipoprotein B from human-plasma low-density lipoproteins. A spin-label study. Eur.J.Biochem. 84: 227-232.

[25] Laggner P, Kostner GM, Rakusch U, Worcester DL (1981) Neutron small-angle scattering on selectively deuterated human plasma low density lipoproteins. The location of polar phospholipid headgroups. J.Biol.Chem. 256, 11832-11839.

[26] Gulik-Krzywicki T, Yates M, Aggerbeck LP (1979) Structure of serum low-density lipoprotein. J.Mol.Biol. 131: 475-484.

[27] Spin JM and Atkinson D (1995) Cryoelectron microscopy of low density lipoprotein in vitreous ice. Biophys.J. 68: 2115-2123.

[28] Laggner P, Chapman MJ, Goldstein S (1978) An X-Ray Small-Angle Scattering Study of Trypsin Treated Low Density Lipoprotein from Human Serum. Biochem.Biophys.Res.Commun. 82: 1332-1339.

[29] Lund-Katz S, Ibdah JA, Letizia JY, Thomas MT, Phillips MC (1988) A ${ }^{13}$ C NMR characterization of lysine residues in apolipoprotein $\mathrm{B}$ and their role in binding to the low density lipoprotein receptor. J.Biol.Chem. 263: 13831-13838.

[30] Prassl, R., Schuster, B., and Laggner, P. (1997) in Supramolecular Structure and Function 5 (Pifat, G., ed.), pp. 47-73, Balaban Publishers.

[31] Prassl R and Laggner P (2009) Molecular structure of low density lipoprotein: current status and future challenges. Eur.Biophys.J.Biophys.Lett. 38: 145-158.

[32] Orlova EV, Sherman MB, Chiu W, Mowri H, Smith LC, Gotto AM (1999) Threedimensional structure of low density lipoproteins by electron cryomicroscopy. Proc.Natl.Acad.Sci.U.S.A 96: 8420-8425.

[33] Van Antwerpen R (2004) Preferred orientations of LDL in vitreous ice indicate a discoid shape of the lipoprotein particle. Arch.Biochem.Biophys. 432: 122-127.

[34] Ren G, Rudenko G, Ludtke SJ, Deisenhofer J, Chiu W, Pownall HJ (2010) Model of human low-density lipoprotein and bound receptor based on cryoEM. Proc Natl Acad Sci U S A 107: 1059-1064.

[35] Kumar V, Butcher SJ, Oorni K, Engelhardt P, Heikkonen J, Kaski K, Ala-Korpela M, Kovanen PT (2011) Three-Dimensional cryoEM Reconstruction of Native LDL Particles to 16 angstrom Resolution at Physiological Body Temperature. PLoS ONE 6.

[36] Liu YH and Atkinson D (2011) Enhancing the Contrast of ApoB to Locate the Surface Components in the 3D Density Map of Human LDL. Journal of Molecular Biology 405: 274-283.

[37] Liu YH and Atkinson D (2011) Immuno-electron cryo-microscopy imaging reveals a looped topology of apoB at the surface of human LDL. J.Lipid Res. 52: 1111-1116.

[38] Deckelbaum RJ, Shipley GG, Small DM, Lees RS, George PK (1975) Thermal transitions in human plasma low density lipoproteins. Science 190, 392-394.

[39] Deckelbaum RJ, Shipley GG, Small DM (1977) Structure and interactions of lipids in human plasma low density lipoproteins. J.Biol.Chem. 252: 744-754.

[40] Laggner P and Müller K (1978) The structure of serum lipoproteins as analysed by Xray small-angle scattering. Q.Rev.Biophys. 11: 371-425. 
[41] Laggner P, Kostner GM, Degovics G, Worcester DL (1984) Structure of the cholesteryl ester core of human plasma low density lipoproteins: Selective deuteration and neutron small- angle scattering. Proc.Natl.Acad.Sci.USA 81: 4389-4393.

[42] Sherman MB, Orlova EV, Decker GL, Chiu W, Pownall HJ (2003) Structure of triglyceride-rich human low-density lipoproteins according to cryoelectron microscopy. Biochemistry 42: 14988-14993.

[43] Coronado-Gray A and Van Antwerpen R (2005) Lipid composition influences the shape of human low density lipoprotein in vitreous ice. Lipids 40: 495-500.

[44] Prassl R, Pregetter M, Amenitsch H, Kriechbaum M, Schwarzenbacher R, Chapman JM, Laggner P (2008) Low density lipoproteins as circulating fast temperature sensors. PLoS ONE 3: e4079.

[45] Liu Y, luo D, Atkinson D (2010) Human LDL core cholesterol ester packing: 3D image reconstruction and SAXS simulation studies. J Lipid Res 51.

[46] Pregetter M, Prassl R, Schuster B, Kriechbaum M, Nigon F, Chapman J, Laggner P (1999) Microphase separation in low density lipoproteins. Evidence for a fluid triglyceride core below the lipid melting transition. J.Biol.Chem. 274: 1334-1341.

[47] Small, D. M. (1986) in The Physical Chemistry of Lipids - From Alkanes to Phospholipids pp. 395-473, Plenum Press, New York and London.

[48] Lusa S and Somerharju P (1998) Degradation of low-density-lipoprotein cholesterol esters by lysosomal lipase in-vitro - effect of core physical state and basis of species selectivity. Bba-Lipid Lipid Metab 1389: 112-122.

[49] Morton RE and Parks JS (1996) Plasma cholesteryl ester transfer activity is modulated by the phase transition of the lipoprotein core. J.Lipid Res. 37: 1915-1923.

[50] Zechner R, Kostner GM, Dieplinger H, Degovics G, Laggner P (1984) In vitro modification of the chemical composition of human plasma low-density lipoproteins: Effects on morphology and thermal properties. Chem.Phys.Lipids 36: 111-119.

[51] Esterbauer H, Dieber-Rotheneder M, Waeg G, Striegl G, Jürgens G (1990) Biochemical, Structural, and Functional Properties of Oxidized Low-Density Lipoprotein. Chem.Res.Toxicol. 3: 77-92.

[52] Chen S-H, Yang C-Y, Chen PF, Setzer D, Tanimura M, Li W-H, Gotto AM, Jr., Chan L (1986) The complete cDNA and amino acid sequence of human apolipoprotein B-100. J.Biol.Chem. 261: 2918-2921.

[53] Knott TJ, Pease RJ, Powell LM, Wallis SC, Rall SC, Innerarity TL, Blackhart B, Taylor WH, Marcel Y, Milne R, Johnson D, Fuller M, Lusis AJ, McCarthy BJ, Mahley RW, LevyWilson B, Scott J (1986) Complete protein sequence and identification of structural domains of human apolipoprotein B. Nature 323: 734-738.

[54] Phillips ML and Schumaker VN (1989) Conformation of apolipoprotein B after lipid extraction of low-density lipoproteins attached to an electron microscope grid. J.Lipid Res. 30: 415-422.

[55] Johs A, Hammel M, Waldner I, May RP, Laggner P, Prassl R (2006) Modular structure of solubilized human apolipoprotein B-100. Low resolution model revealed by small angle neutron scattering. J.Biol.Chem. 281: 19732-19739. 
[56] Chatterton JE, Phillips ML, Curtiss LK, Milne RW, Marcel YL, Schumaker VN (1991) Mapping apolipoprotein B on the low density lipoprotein surface by immunoelectron microscopy. J.Biol.Chem. 266: 5955-5962.

[57] Chatterton JE, Schlapfer P, Bütler E, Gutierrez MM, Puppione DL, Pullinger CR, Kane JP, Curtiss LK, Schumaker VN (1995) Identification of apolipoprotein B 100 Polymorphisms that affect low-density lipoprotein metabolism: Description of a new approach involving monoclonal antibodies and dynamic light scattering. Biochemistry 34: 9571-9580.

[58] Mann CJ, Anderson TA, Read J, Chester SA, Harrison GB, Kochl S, Ritchie PJ, Bradbury P, Hussain FS, Amey J, Vanloo B, Rosseneu M, Infante R, Hancock JM, Levitt DG, Banaszak LJ, Scott J, Shoulders CC (1999) The structure of vitellogenin provides a molecular model for the assembly and secretion of atherogenic lipoproteins. J.Mol.Biol 285: 391-408.

[59] Wang L, Walsh MT, Small DM (2006) Apolipoprotein B is conformationally flexible but anchored at a triolein/water interface: a possible model for lipoprotein surfaces. Proc.Natl.Acad.Sci.U.S.A 103: 6871-6876.

[60] Wang L, Martin DD, Genter E, Wang J, McLeod RS, Small DM (2009) Surface study of apoB1694-1880, a sequence that can anchor apoB to lipoproteins and make it nonexchangeable. J Lipid Res 50: 1340-1352.

[61] Mikl C, Peters J, Trapp M, Kornmueller K, Schneider WJ, Prassl R (2011) Softness of atherogenic lipoproteins: a comparison of very low density lipoprotein (VLDL) and low density lipoprotein (LDL) using elastic incoherent neutron scattering (EINS). J Am Chem Soc 133: 13213-13215.

[62] Lobatto ME, Fuster V, Fayad ZA, Mulder WJM (2011) Perspectives and opportunities for nanomedicine in the management of atherosclerosis. Nature Reviews Drug Discovery 10: 835-852.

[63] Duncan R and Gaspar R (2011) Nanomedicine(s) under the Microscope. Molecular Pharmaceutics 8: 2101-2141.

[64] Allen TM and Cullis PR (2004) Drug delivery systems: entering the mainstream. Science 303: 1818-1822.

[65] Ng KK, Lovell JF, Zheng G (2011) Lipoprotein-Inspired Nanoparticles for Cancer Theranostics. Accounts of chemical research 44: 1105-1113.

[66] Frias JC, Lipinski MJ, Lipinski SE, Albelda MT (2007) Modified lipoproteins as contrast agents for imaging of atherosclerosis. Contrast.Media Mol.Imaging 2: 16-23.

[67] Cormode DP, Skajaa T, Fayad ZA, Mulder WJ (2009) Nanotechnology in medical imaging: probe design and applications. Arterioscler Thromb Vasc Biol 29: 992-1000.

[68] Hammel M, Laggner P, Prassl R (2003) Structural characterisation of nucleoside loaded low density lipoprotein as a main criterion for the applicability as drug delivery system. Chem.Phys.Lipids 123: 193-207.

[69] Song LP, Li H, Sunar U, Chen J, Corbin I, Yodh AG, Zheng G (2007) Naphthalocyaninereconstituted LDL nanoparticles for in vivo cancer imaging and treatment. International Journal of Nanomedicine 2: 767-774. 
[70] Corbin IR, Li H, Chen J, Lund-Katz S, Zhou R, Glickson JD, Zheng G (2006) Lowdensity lipoprotein nanoparticles as magnetic resonance imaging contrast agents. Neoplasia 8: 488-498.

[71] Chen LC, Chang CH, Yu CY, Chang YJ, Hsu WC, Ho CL, Yeh CH, Luo TY, Lee TW, Ting G (2007) Biodistribution, pharmacokinetics and imaging of Re-188-BMEDAlabeled pegylated liposomes after intraperitoneal injection in a C26 colon carcinoma ascites mouse model. Nuclear Medicine and Biology 34: 415-423.

[72] Zheng G, Chen J, Li H, Glickson JD (2005) Rerouting lipoprotein nanoparticles to selected alternate receptors for the targeted delivery of cancer diagnostic and therapeutic agents. Proc.Natl.Acad.Sci.U.S.A 102: 17757-17762.

[73] Zhang ZH, Chen J, Ding LL, Jin HL, Lovell JF, Corbin IR, Cao WG, Lo PC, Yang M, Tsao MS, Luo QM, Zheng G (2010) HDL-Mimicking Peptide-Lipid Nanoparticles with Improved Tumor Targeting. Small 6: 430-437.

[74] Prassl R, Chapman JM, Nigon F, Sara M, Eschenburg S, Betzel C, Saxena A, Laggner P (1996) Crystallization and preliminary X-ray analysis of a low density lipoprotein from human plasma. J.Biol.Chem. 271: 28731-28733. 